\title{
Avaliação da atividade antimicobacteriana da lignana diidrocubebina extraída da Piper cubeba e de seus derivados semissintéticos
}

\author{
LAURENTIZ, R.S. ${ }^{*}$; BORGES, A."; LAURENTIZ, A.C.'; SILVA, M.L.A. ${ }^{2}$, MARTINS, C.H.G. ${ }^{2}$ \\ ${ }^{1}$ Departamento de Física e Química, Faculdade de Engenharia de Ilha Solteira, Universidade Estadual \\ Paulista, Avenida Brasil 56, Ilha Solteira, 15385-000, SP, Brazil. "2Núcleo de Ciências Exatas e Tecnológicas, \\ Universidade de Franca, Avenida Dr. Armando Salles de Oliveira 2001, 14404-600, Franca, SP, Brazil. *Autor \\ para correspondência: rosangela@dfq.feis.unesp.br
}

\begin{abstract}
RESUMO: Aatividade antimicobacteriana de diidrocubebina (1), uma lignana dibenzilbutanodioica obtida a partir de extrato etanólico de sementes da Piper cubeba, e seus derivados foram avaliados in vitro contra três diferentes cepas de Mycobacterium utilizando o método de microdiluição. Dentre as lignanas avaliadas 3 e 4 foram as mais ativas, exibindo valores de CIM de $62,5 \mu \mathrm{g} / \mathrm{mL}$ contra $M$. avium e M. tuberculosis, respectivamente. Os derivados 2-6 obtidos por síntese parcial possuem diferentes substituintes nos carbonos 9 e 9 ', que alteram polaridade, solubilidade e limitam as rotações livres entre C8-C8' em relação de material (1) de partida. As diferenças estruturais entre estes compostos podem fornecer informações importantes sobre a relação estrutura-atividade antimicobacteriana do esqueleto dibenzilbutanodioico, obtido a partir de fonte natural, como um possível alvo para o desenvolvimento de drogas mais potentes contra a tuberculose.
\end{abstract}

Palavras-chave: lignanas, diidrocubebina, atividade antimicobacteriana, Piper cubeba

\begin{abstract}
Evaluation of antimycobacterial activity of dihydrocubebin lignan extracted from Piper cubeba and its semisynthetic derivatives. The antimycobacterial activity of the dihydrocubebin (1), a dibenzylbutanedioiclignan obtained from ethanolic extract of Piper cubeba seeds, and its derivatives were examined in vitro against three different strains of Mycobacterium using amicrodilution method. Among the lignans evaluated, the $\mathbf{3}$ and $\mathbf{4}$ samples were the most active ones, displaying MIC values of $62.5 \mu \mathrm{g} / \mathrm{mL}$ against $M$. avium and M. tuberculosis, respectively. The derivatives 2-6, obtained for partial synthesis, had different substituents in the carbons 9 and ' ', fact thatalters the polarity, solubility and restricts the free rotations between the bonds C8-C8' in relation to the starting material (1). The structural differences among these compounds provide important information about the antimycobacterial structure-activity relationship of the dibenzylbutanodioic skeleton, obtained from natural source, such as a possible target for the development of more powerful drugs against tuberculosis.
\end{abstract}

Keywords: lignan, dihydrocubebin, antimycobacterial activity, Piper cubeba

\section{INTRODUÇÃO}

A tuberculose (TB) é uma doença infecciosa grave altamente contagiosa e um dos principais problemas de saúde pública atualmente (Harris et al., 2014). De acordo com a WHO (2014), em 2012 foram estimados mais de 9,3 milhões de novos casos de TB no mundo e 1,3 milhões de óbitos provocados pela doença. Sem um controle mais efetivo, cerca de 1,5 bilhões de pessoas serão infectadas e 3,5 milhões morrerão até 2020. O tratamento clínico para a TB inclui a combinação de drogas como rifampicina, isoniazida e pirazinamida.
Apesar desse tratamento ser efetivo, o uso incorreto, longos períodos de administração, bem como alto custo e sérios efeitos colaterais fez ao longo dos anos com que muitos pacientes abandonassem 0 tratamento antes da cura completa o que culminou no surgimento de bacilos multi resistentes (Hardman et al., 2001; Timmins \& Deretic, 2006; Arbex et al., 2010; Diacon et al, 2014; Harris et al., 2014). Embora existam inúmeros programas governamentais e estratégias para o controle da TB, a descoberta de novas drogas pode ser uma das alternativas para 
auxiliar no controle da doença (Raviglione, 2003; Okunade \& Elvin-Lewis, 2004; Koul et al., 2011; Riccardi \& Pasca, 2014).

Neste sentido, a necessidade de novos agentes antimicrobianos que apresentem menos efeitos colaterais e menor tempo de tratamento tem levado pesquisadores a focarem suas pesquisas em moléculas de ocorrência natural assim como tem sido feito para muitas outras doenças (Verg et al., 1998, Damayanthi \& Lown 1998; Gordaliza et al., 2004; Akinyemi et al., 2005; Dey et al., 2014).

Garcí et al. (2012) publicaram um artigo de revisão sobre produtos naturais com atividade in vitro sobre cepas sensíveis e resistentes de Mycobacterium. Esta revisão inclui produtos naturais estruturalmente relevantes com CIM a partir de $128 \mu \mathrm{g} / \mathrm{mL}$ e análogos sintéticos com considerável atividade antimicobacteriana (CIM $\leq 10 \mu \mathrm{g} / \mathrm{mL})$ e dentre esses compostos alguns deles são lignanas.

Lignanas são metabólitos secundários produzidos pelas plantas que apresentam grande diversidade estrutural e geralmente estão associados à defesa da planta contra a ação de patógenos, ocorrem em várias espécies de plantas sendo frequentes no gênero Piper. Piper cubeba é uma pimenta asiática utilizada na medicina tradicional disenteria na Indonésia para o tratamento de gonorreia, sífilis, enterites, e dores abdominais, (Elfahmi et al., 2007). P. cubeba contém várias lignanas com diferentes estruturas químicas cujos constituintes de maior ocorrência são a hinoquinina, cubebina e yateína às quais tem sido atribuída muitas das propriedades medicinais da P. cubeba. (Timple et al., 2013; Usia et al., 2005; Yam et al., 2008a; 2008b). No entanto, a P. cubeba apresenta outra lignana interessante (Elfahmi et al., 2007), a diidrocubebina (1), que devido à sua estrutura química permite a realização de inúmeras

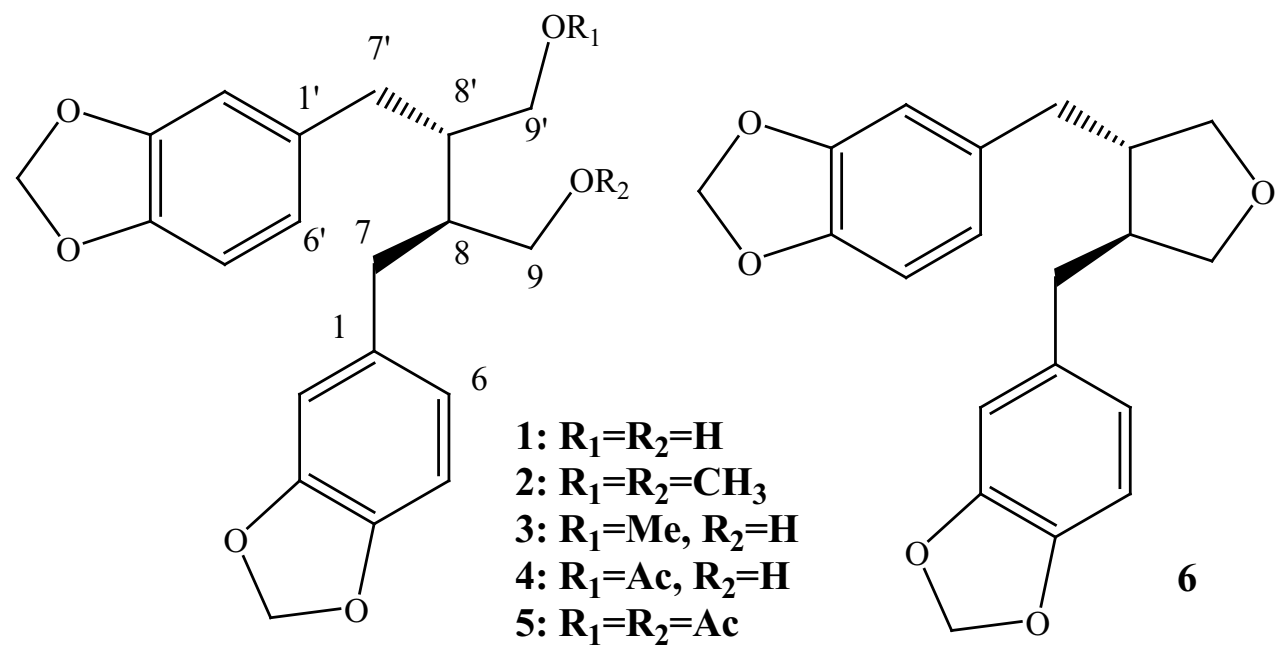

modificações estruturais com a obtenção de derivados que podem ser úteis para a descoberta de novas drogas.

Em nossos estudos anteriores sobre as lignano lactonas e lactóis várias propriedades biológicas foram relatadas (Saraiva et al., 2007; 2009, Silva et al., 2009, Silva et al., 2005; Silva et al., 2008; Souza et al., 2005; Silva et al., 2011; Pistolozzi et al., 2013), incluindo a atividade antimicobacteriana (Silva et al., 2009). Portanto, para estender nossas investigações sobre esta importante classe de produtos naturais, a atividade antimicobacteriana da diidrocubebina (1), uma lignana dibenzilbutanodioica, e seus derivados (Figura 1) foram avaliados contra M. tuberculosis, M. kansasii e M. avium.

\section{MATERIAL E METODOS Materiais e equipamentos}

Os solventes utilizados nas extrações e nas purificações por cromatografia em coluna possuíam grau analítico e àqueles utilizados nas sínteses foram adquiridos da Sigma-Aldrich. Os espectros de RMN foram obtidos num espectrômetro Bruker ARX 400 em $\mathrm{CDCl}_{3}$ a $400 \mathrm{MHz}$ para ${ }^{1} \mathrm{H}$ RMN e 100 $\mathrm{MHz}$ para ${ }^{13} \mathrm{C}$ RMN usando ressonância residual do $\mathrm{CDCl}_{3}$ como referência interna. CG-MS foram registrados em um espectrômetro Shimadzu GCMS QP2010 com injetor AOC-20i automático. As cromatografias em camada delgada (CCD) foram realizadas empregando-se placas de alumínio recobertas de sílica gel $60 \mathrm{~F}_{254}$ com espessura de $0,25 \mathrm{~mm}$ (Merck) utilizando misturas de Hex/AcOEt como solvente.

\section{Material Vegetal}

Sementes de $P$. cubeba foram adquiridos da empresa Floral Seed Company, Dehradun, Índia.

FIGURA 1. Estrutura química das lignanas 1-6 


\section{Extração e Isolamento}

As sementes de $P$. cubeba foram secas em estufa de circulação de ar a $40^{\circ} \mathrm{C}$ e pulverizadas em moinhos de facas (Marconi). Foram utilizados $2,0 \mathrm{Kg}$ do material pulverizado para o processo de extração o quais foram imersos em $6 \mathrm{~L}$ de etanol à temperatura ambiente em um frasco de vidro, durante 7 dias, após este período, o extrato foi filtrado e concentrado em vácuo. Este processo foi repetido por mais 3 vezes. Os extratos foram combinados para fornecer $400 \mathrm{~g}$ de extrato etanólico seco (EE).

O extrato etanólico (EE) foi suspenso em solução hidrometanólica (9:1), para cada $20 \mathrm{~g}$ de EE foram utilizados aproximadamente $100 \mathrm{~mL}$ de solução hidrometanólica. Esta solução foi submetida a partição com hexano $(7 \times 250 \mathrm{~mL})$, e em seguida separada e concentrada. Este procedimento forneceu $90 \mathrm{~g}$ (fração hidrometanólica), dos quais $45 \mathrm{~g}$ foram adicionados numa cápsula de porcelana contendo de etanol e sílica gel. Em seguida, a cápsula de porcelana, com o extrato incorporado na sílica, foi mantida em banho-maria, com temperatura não superior à $55^{\circ} \mathrm{C}$, para a eliminação do solvente. A pastinha obtida foi aplicada a uma coluna de sílica gel eluída, sequencialmente, com diferentes sistemas de solventes: Hex, Hex/DCM, DCM/AcOEt e AcOEt.

Frações de $400 \mathrm{~mL}$ foram coletadas e concentradas. Este processo de coleta de frações foi monitorado por CCDC. Depois de reveladas as placas cromatográficas com vanilina sulfúrica, seus perfis cromatográficos foram comparados, sendo que as subfrações semelhantes foram reunidas, resultando em 20 frações (Tab. 1).

Das 20 frações resultantes, aquelas com padrões similares de CCD foram combinadas fornecendo 8 frações principais [A (3,81 g), B (6,49 g), C (9,60 g), D (20,5 g), E (8,0 g), F (5,2 g), L $(0,84 \mathrm{~g}), \mathrm{H}(1,52 \mathrm{~g})]$. A fração $E$ foi novamente submetida a cromatografia em coluna de sílica gel utilizando como eluente Hex seguido pela mistura Hex/AcOEt (9:1) em gradiente crescente de AcOEt até AcOEt puro. Frações de $50 \mathrm{~mL}$ foram coletadas e concentradas. Este processo de coleta de frações foi monitorado por CCD sendo as frações com perfis cromatográficos semelhantes reunidas totalizando 10 subfrações.

As frações eluídas com a mistura de Hex/AcOEt na proporção 1:1 foram reunidas, concentradas e o resíduo sólido formado foi recristalizado com a mistura de Hex/DCM 7:3 para a obtenção de um composto cristalino $(500,2 \mathrm{mg}$, Rf 0,31).

Os dados de RMN e CG-MS obtidos para esse composto estão de acordo com os relatados na literatura para a lignana diidrocubebina (1) (Badheka et al., 1987).

Diidrocubebina (1): Sólido incolor pf $102^{\circ} \mathrm{C}$. IR $(\mathrm{KBr}): \mathrm{v}=3430,3337,2340,1700,1650,1505$, 1246, 1036. ${ }^{1} \mathrm{H} \mathrm{RMN} \mathrm{d}\left(\mathrm{CDCl}_{3}\right): 6,6(\mathrm{~m}, 6 \mathrm{H}) ; 5,8$ (s,

TABELA 1. Subfrações obtidas do fracionamento da FHM do EE.

\begin{tabular}{|c|c|c|c|}
\hline Subfrações & Frações & Massa $(g)$ & Eluente (v/v) \\
\hline 01 a 02 & 1 & 0,05 & Hexano \\
\hline 03 a 06 & 2 & 0,30 & Hexano \\
\hline 07 a 24 & 3 & 3,35 & Hex/DCM 9:1 \\
\hline 25 a 32 & 4 & 0,39 & Hex/DCM 8:2 \\
\hline 33 a 34 & 5 & 0,27 & Hex/DCM 8:2 \\
\hline 35 a 39 & 6 & 2,65 & Hex/DCM 7:3 \\
\hline 40 a 42 & 7 & 1,63 & Hex/DCM 7:3 \\
\hline 43 a 49 & 8 & 2,03 & Hex/DCM 7:3 \\
\hline 50 a 68 & 9 & 9,15 & Hex/DCM 6:4/1:1 \\
\hline 69 a 79 & 10 & 21,03 & DCM/AcOEt 8:2 \\
\hline 80 a 84 & 11 & 8,15 & DCM/AcOEt 8:2 \\
\hline 85 a 90 & 12 & 6,15 & DCM/AcOEt 8:2 \\
\hline 91 a 92 & 13 & 0,91 & DCM/AcOEt 7:3 \\
\hline 93 a 97 & 14 & 0,94 & DCM/AcOEt 6:4 \\
\hline 98 a 99 & 15 & 0,33 & DCM/AcOEt 6:4 \\
\hline 100 a 102 & 16 & 0,60 & DCM/AcOEt 1:1 \\
\hline 103 a 105 & 17 & 0,51 & DCM/AcOEt 1:1 \\
\hline 106 a 107 & 18 & 0,45 & DCM/AcOEt 1:1 \\
\hline 108 & 19 & 0,15 & AcOEt \\
\hline $109-115$ & 20 & 0,63 & AcOEt \\
\hline
\end{tabular}

Rev. Bras. PI. Med., Campinas, v.17, n.4, supl. I, p.782-789, 2015. 
4H); 3,7 (dd, 2H, J=1,3 e 11,3 Hz); 3,4 (dd, 2H, J= 4,1 e 11,3 Hz); 3,2 (s.l, 2H); 2,7 (d, 1H, J=8,7 Hz); 2,65 (d, 1H, J= 8,7 Hz); 2,56 (d, 1H, J=5,7 Hz); 2,51 (d, 1H, J=5,7 Hz); 1,75 (m, 2H). ${ }^{13} \mathrm{C}$ RMN d $\left(\mathrm{CDCl}_{3}\right)$ :147,6;145,7; 134,3; 121,9; 109,3; 108,1; 100,8; 60,2; 44,3; 35,9. EM m/z: 358 (10) $\mathrm{M}^{+}, 340$ (15), 203 (22), 187 (14), 172 (8), 160 (6), 136 (35), 135 (100), 77 (10).

\section{Síntese Parcial}

Síntese da diidrocubebina dimetil éter (2) e diidrocubebina monometil éter (3).

A uma solução de 1 (100 mg) em THF anidro (5 mL), sob $\mathrm{N}_{2}$ e agitação, foi adicionada uma suspensão de $\mathrm{NaH}$ (1,2 mg em $2 \mathrm{~mL}$ de THF). Essa mistura foi mantida sob agitação à temperatura ambiente $\left(28^{\circ} \mathrm{C}\right)$ durante 30 min e em seguida foram adicionados $0,5 \mathrm{~mL}$ de $\mathrm{CH}_{3}$. A mistura foi agitada por mais $6 \mathrm{~h}$ e após este período foram adicionados 5 $\mathrm{mL}$ de água seguidos pela extração com EtOAc (3 $\times 15 \mathrm{~mL}$ ). A fase orgânica foi separada, seca sobre $\mathrm{MgSO}_{4}$, e filtrada. O solvente foi evaporado sob pressão reduzida, o resíduo produzido foi purificado por cromatografia em coluna de sílica gel usando n-Hex/EtOAc (9:1) como eluente fornecendo os compostos 2 (62,5 mg) e 3 (20,8 mg). Os dados de RMN e CG-MS obtidos para o composto 2 estão de acordo com os reportados na literatura (Badheka et al., 1987).

Diidrocubebina dimetil éter (2): ${ }^{1} \mathrm{H}$ RMN d $\left(\mathrm{CDCl}_{3}\right)$ 6,6 (d, 2H, J = 8.0 Hz); 6,5 (s.l, 2H); 6,5 (d, $2 \mathrm{H}, \mathrm{J}=8.0 \mathrm{~Hz}) ; 5,8$ (s, 4H); 3,23 (dd, 2H, J=5,7 e 9,5 Hz); 3,21 (s, 6H); 3,19 (dd, 2H, J=4,9 e 9,5 Hz); 2,58 (dd, 2H, J= 6,7 e 13,6 Hz); 2,48 (dd, 2H, J= 8,2 e 13,6 HZ); 1,98 (m, 2H). ${ }^{13} \mathrm{C}$ RMN d $\left(\mathrm{CDCl}_{3}\right): 147,5$; 145,6; 134,9; 121,8; 109,5; 108,5; 108,1; 100,7; 72,6; 58,8; 40,7; 35,1. EM m/z: $386\left(\mathrm{M}^{+}\right)(2), 187$ (19), 135 (100), 77 (29).

Diidrocubebina monometil éter (3): ${ }^{1} \mathrm{H}$ RMN d $\left(\mathrm{CDCl}_{3}\right): 6,6$ (d, 2H, J = 7,7, Hz); 6,5 (sl, 2H); 6,4 (d, 2H, J=7,7 Hz); 5,8 (s, 4H); 3,6-3,4 (m, 2H); 3,3 (dd, $1 \mathrm{H}, J=5,7$ e 9,5 Hz); 3,2 (s, 3H); 3,2 (dd, 1H, J = 4,9 e 9,5 Hz); 2,5 (d, 2H, J = 6,7 e 13,6 Hz); 2,4 (dd, $2 \mathrm{H}, \mathrm{J}=8,2$ e 13,6 Hz); 2,0 (m, 2H); 1,8 (sl, $1 \mathrm{H}) .{ }^{13} \mathrm{C}$ RMN d $\left(\mathrm{CDCl}_{3}\right): 147,2 ; 145,31 ; 134,67 ; 121,9 ; 109,3$; 108,$3 ; 108,1 ; 100,8 ; 72,4 ; 65,6 ; 58,6 ; 40,9 ; 34,9$. EM m/z: $372(\mathrm{M})^{+}(12), 354$ (20), 323 (25), 135 (100).

\section{Síntese da Hemiariensina (4) e Ariensina (5)}

Diidrocubebina (1) $(50,0 \mathrm{mg})$ foi adicionada a uma solução contendo $\mathrm{Et}_{3} \mathrm{~N}(0,08 \mathrm{~mL}), \mathrm{Ac}_{2} \mathrm{O}(0,04$ $\mathrm{mL})$, DMAP (2 mg), e THF (1 mL). Esta mistura foi mantida sob agitação por $3 \mathrm{~h}$. Os derivados, monoacetilado 4 (14,1 mg) e diacetilado 5 (30,6 mg), foram obtidos após usual "quench" e purificação em coluna de sílica gel (Hex/EtOAc 8:2). Os dados de RMN e CG-MS obtidos para os compostos 4 e 5 estão de acordo com os reportados na literatura (Badheka et al., 1987; Hernandez et al., 1983). Hemiaresina (4): $1 \mathrm{H}$ RMN d $\left(\mathrm{CDCl}_{3}\right): 6,8-6,5$ (m, 6H); 5,9 (s, 4H); 4,1 (m, 2H); 3,6 (d, 2H, J = 8,0 $\mathrm{Hz}) ; 2,6-2,4$ (m, 4H); 2,1 (s, 3H); 2,05 (m, 2H); 1,6 (s.l, 1H). ${ }^{13} \mathrm{C}$ RMN (75 MHz, $\mathrm{CDCl}_{3}$ ): 170,3; 148,6; 146,$1 ; 132,1 ; 121,9 ; 121,5 ; 109,5 ; 108,2 ; 108,8$; 107,$5 ; 100,8 ; 67,8 ; 65,2 ; 45,1 ; 39,5 ; 35,6 ; 35,3 ; 20,8$. EM m/z: 400 (M)+ (50), 340 (7), 204 (15), 187 (25), 179 (8), 161 (11), 136 (32), 135 (100).

Ariensina (5): ${ }^{1} \mathrm{H} \mathrm{RMN} \mathrm{d}\left(\mathrm{CDCl}_{3}\right)$ 6,6 (d, 2H, $J=7,70 \mathrm{~Hz}) ; 6,5$ (m, 4H); 5,9 (s, 4H); 4,0 (dd, 2H, J $=5,90$ e 11,3 Hz); 3,9 (dd, $2 \mathrm{H}, J=4,90$ e 11,1 Hz); 2,6 (dd, $2 \mathrm{H}, J=6,20$ e 13,7 Hz); 2,5 (dd, $2 \mathrm{H}, J=$ $8,0$ e $14,0 \mathrm{~Hz}) ; 2,0$ (m, 2H); 2,0 (s, 6H). ${ }^{13} \mathrm{C}$ RMN d $\left(\mathrm{CDCl}_{3}\right): 170,3 ; 147,5 ; 145,6 ; 133,1 ; 121,8 ; 108,8$; 107,$5 ; 100,7 ; 64,2 ; 40,1 ; 39,1 ; 35,0 ; 20,5$.

\section{Síntese da Desidroxicubebina (6)}

Uma solução de 1 (50 mg) em diclorometano $(2 \mathrm{~mL})$ foi adicionada a uma solução de APTS (24 mg) em diclorometano (8 mL), a mistura resultante foi mantida sob agitação por $12 \mathrm{~h}$ a temperatura ambiente $\left(28^{\circ} \mathrm{C}\right)$. Após este período foram adicionados $5 \mathrm{~mL}$ de água seguido pela extração com DCM $(3 \times 10 \mathrm{~mL})$. A fase orgânica foi separada, seca sobre $\mathrm{MgSO}_{4}$, e filtrada. O solvente foi evaporado sob pressão reduzida, o resíduo produzido foi purificado por cromatografia em coluna de sílica gel usando Hex/EtOAc (9:1) como eluente, fornecendo o composto 6 (42,8 $\mathrm{mg})$. Os dados de RMN e CG-MS obtidos para o composto 6 estão de acordo com os reportados na literatura (Carvalho et al., 1987).

Desidroxicubebina (6): ${ }^{1} \mathrm{H}$ RMN d $\left(\mathrm{CDCl}_{3}\right)$ : 6,65 (d, 2H, J= 8,0 Hz); 6,48 (sl, 2H); 6,46 (dl, 2H, $\mathrm{J}=8,0 \mathrm{~Hz}$ ); 5,85 (s, 4H); 3,82 (dd, 2H, J= 6,7 e 8,8 $\mathrm{Hz}$ ); 3,42 (dd, 2H, J=5,9 e 8,8 Hz); 2,50 (dd, 2H, J= 6,2 e13,6 Hz); 2,42 (dd, 2H, J= 8,0 e 13,6 Hz); 2,07 (m, 2H). ${ }^{13} \mathrm{C} \mathrm{RMN} \mathrm{d}\left(\mathrm{CDCl}_{3}\right): 147,5 ; 145,7 ; 133,9$; 121,3; 108,1; 108,7; 100,7; 72,9; 46,5; 39,1. MS m/z: $340\left(\mathrm{M}^{+}\right)(25), 135$ (100), $122(5), 77(10)$.

\section{Atividade antimicobacteriana}

A atividade antimicobacteriana dos compostos 1-6 foi avaliada in vitro por meio da técnica de microdiluição em microplaca utilizando resazurina como indicador de atividade microbiana (REMA- Resazurin Microtiter Assay), (Palomino et al., 2002), o que permitiu a determinação da concentração inibitória mínima (CIM) contra o Mycobacterium tuberculosis H37Rv (ATCC 27294), M. kansasii (ATCC 12478) e M. avium (ATCC 15769). Os compostos foram dissolvidos em dimetil-sulfóxido (DMSO) e diluídos em série em caldo Middlebrook 7H9 antes da inoculação. As concentrações dos 
compostos testados variaram de 15 a $2000 \mu \mathrm{g} /$ $\mathrm{mL}$, sendo a concentração final de DMSO menor que $0,3 \%$. Rifampicina (Sigma) foi usada como o antibiótico de referência nas concentrações de 0,015 a $1,0 \mu \mathrm{g} / \mathrm{mL}$. O caldo Middlebrook $7 \mathrm{H} 9$ e o inóculo foram usados como controle negativo e positivo, respectivamente. A microplaca foi selada com parafilme e incubada a $37^{\circ} \mathrm{C}$. Após sete dias de incubação, $30 \mu \mathrm{L}$ de uma solução aquosa de resazurina a $0,01 \%$ (Sigma-Aldrich) diluída em água esterilizada foi adicionada. A microplaca foi reincubada a $37^{\circ} \mathrm{C}$ por 24 horas, seguida da leitura colorimétrica visual. Os ensaios foram realizados em triplicatas. Os valores de CIM foram definidos como a menor concentração da droga capaz de inibir o crescimento bacteriano.

\section{RESULTADOS E DISCUSSÃO}

Isoniazida e rifampicina são as drogas mais eficazes contra a TB e foram descobertas na década de 1950 e 1960, desde então o tratamento para a doença vem sendo feito em muitos países pela combinação dessas duas drogas. Apesar dessas duas drogas serem eficazes no tratamento da doença muitos pacientes abandonam seu uso devido ao longo período de tratamento (em média 6 meses) ao qual o paciente sofre com sérios efeitos colaterais das drogas (Riccardi \& Pasca, 2014). O abandono do tratamento sem a obtenção da cura contribuiu para o surgimento de cepas de Mycobacterium resistentes a essas drogas o que agrava ainda mais o quadro da doença.

Com o surgimento da resistência do Mycobacterium às essas drogas surgiu a necessidade de se buscar novas drogas mais eficazes e com menor tempo de tratamento. A busca por novas fontes de moléculas que possam ser precursoras de fármacos contra a TB passou então a ser um dos promissores caminhos alternativos para busca ao tratamento da doença.

Entretanto, não existe o interesse por parte da indústria farmacêutica no sentido de investir grandes recursos no desenvolvimento de novas drogas contra a TB devido à sua predominância em países subdesenvolvidos. Por milhares de anos as plantas, através de seus metabólitos secundários, têm sido utilizadas para o tratamento de inúmeras doenças principalmente por populações carentes em vários países inclusive para o tratamento da TB. Neste sentido inúmeras plantas usadas na medicina tradicional já foram avaliadas quanto ao seu potencial antimicobacteriano na forma de extratos e depois de seus principais constituintes isoladamente como uma forma alternativa na busca por drogas mais potentes e com menos efeitos colaterais do que as utilizadas atualmente.
A gravidade da doença aliada a existência de cepas de Mycobacterium resistentes reforça a importância e a necessidade de pesquisar novas fontes de moléculas bioativas para o desenvolvimento de novas drogas mais efetivas contra a TB (Harris et al., 2014).

Visando avaliar lignanas como novas fontes de moléculas contra TB a atividade antimicobacteriana dos compostos $\mathbf{1}$ - $\mathbf{6}$ foi determinada através da concentração inibitória mínima (CIM) usando Rifampicina como droga de referência (Collins e Franzblau, 1997). A Rifampicina é um antibiótico que atua bloqueiando a transcrição bacteriana através da inibição da ARN-polimerase (Walsh, 2003). Apesar de eficaz já existem cepas de micobactérias resistentes a esse antibiótico, sendo que o surgimento da resistência se deve ao desenvolvimento de mutações na subunidade $B$ da RNA polimerase do bacilo que tornam incapaz a enzima de fixar o antibiótico (Dzidic, et al., 2008; Guimarães et al., 2010).

Os resultados da avaliação dos compostos 1-6 são apresentados na tabela 1. Os compostos avaliados apresentaram altos valores de CIM $(2000 \mu \mathrm{g} / \mathrm{mL})$ contra o M. kansasii o que os desqualificam como moléculas promissoras para o desenvolvimento de novos fármacos contra essa cepa (Garcí et al., 2012). Todas as cepas utilizadas foram sensíveis ao controle positivo Rifampicina com valores de CIM extremamente baixas (Tabela 1), mas diferentes entre as três cepas de micobactérias avaliadas.

As micobactérias possuem a parede celular hidrofóbica constituída por diferentes lipídios que diferem também entre várias as espécies de micobactérias (Korycka-Machala et al., 2005). Desta forma, para que um composto possa exercer um efeito tóxico sobre as diferentes cepas de micobactéria inicialmente ele tem que penetrar a membrana celular do bacilo através da barreira de lipídios da parede celular ou pelas porinas no caso de compostos hidrofílicos. A forma com que os compostos fazem a penetração na micobactéria depende das características intrínsecas de cada molécula (Declour, 2009; Moreira et al., 2013). Portanto, a baixa atividade dos compostos contra $M$. kansasii pode estar relacionada a incapacidade desses compostos de penetrar a membrana celular dessa cepa (Guimarães et al., 2010; Murray et al., 2012)

Em relação às outras cepas avaliadas os compostos que apresentaram maior atividade, com CIM = 62,5 $\mu \mathrm{g} / \mathrm{mL}$, foram a lignana 3 contra a $M$. tuberculosis e a lignana 4 contra $M$. avium. Os demais compostos apresentaram CIM de 250 ou $125 \mu \mathrm{g} / \mathrm{mL}$ para essas duas cepas. Existem muitos trabalhos na literatura sobre a avaliação

Rev. Bras. PI. Med., Campinas, v.17, n.4, supl. I, p.782-789, 2015. 
TABELA 2. Concentração inibitória mínima (CIM) de lignanas 1-6 contra o M. tuberculosis, M. kansasii e M. avium

\begin{tabular}{cccc}
\hline \multirow{2}{*}{ Compostos } & \multicolumn{3}{c}{ Concentração Inibitória Mínima $(\boldsymbol{\mu g} / \mathbf{m L})$} \\
& M. kansasii & M. avium \\
(ATCC 27294) & (ATCC 12478) & (ATCC 15769) \\
\hline $\mathbf{1}$ & $125 \pm$ & 2000 & 125 \\
$\mathbf{2}$ & 250 & 2000 & 250 \\
$\mathbf{3}$ & 62,5 & 2000 & 125 \\
$\mathbf{4}$ & 125 & 1000 & 62,5 \\
$\mathbf{5}$ & 250 & 2000 & 250 \\
$\mathbf{6}$ & 125 & 2000 & 250 \\
Rifampicina $^{a}$ & 0,031 & 0,015 & 0,062 \\
\hline
\end{tabular}

aAntibiótico usado como controle positivo

antimicobacteriana de compostos naturais ou sintético, entretanto, os valores de CIM que definem o grau de atividade desses compostos divergem entre as publicações (Cantrell et al., 2001; Gu et al., 2004; Garcí et al., 2012).

Para Cantrell et al. (2001) compostos isolados que apresentam CIM de $64 \mu \mathrm{g} / \mathrm{mL}$ ou menor são considerados promissores. Segundo Gu et al. (2004) CIM $\leq 128 \mu \mathrm{g} / \mathrm{mL}$ definem uma substância como ativa contra M. tuberculosis. Garcí et al. (2012) relataram substâncias como ativas com CIM $\leq 128$ $\mu \mathrm{g} / \mathrm{mL}$, sendo que quanto menor o valor de CIM mais ativa é a substância.

Portanto, de acordo com os dados de Gu et al. (2004) e Garcí et al (2012) a lignana 1 que apresentou $\mathrm{CIM}=125 \mu \mathrm{g} / \mathrm{mL}$ contra as duas cepas e a lignana $6 \mathrm{com} \mathrm{CIM}=125 \mu \mathrm{g} / \mathrm{mL}$ contra M. tuberculosis podem também ser consideradas ativas. No entanto, apesar de manter o mesmo CIM do composto 1 a modificação estrutural para a obtenção de 6 não contribuiu para o aumento de atividade.

Acomparação das estruturas dos compostos que apresentaram maior atividade nos ensaios in vitro mostra que o composto 3 difere do 4 apenas pela presença do grupo metoxila cuja posição no composto 4 é ocupada por um grupo acetila. Essa diferença estrutura também proporciona diferença de atividade contra as cepas $M$. tuberculosis e M. avium. Esses compostos possuem natureza hidrofóbica, o que pode indicar, com base na lipofilicidade, que o composto 3 consegue penetrar na barreira de lipídios da cepa $M$ tuberculosis mais facilmente do que na cepa $M$. avium. O mesmo pode-se dizer sobre a atividade do composto $4 \mathrm{em}$ relação à cepa $M$. avium.

Na diidrocubebina a presença da segunda hidroxila diminui a lipofilicidade do composto o que pode dificultar a penetração através da barreira lipídica contribuindo para a menor atividade apresentada por essa lignana. Entretanto, ao se comparar a estrutura dos compostos 2 e 5 com 3 e
4 é possível verificar que as diferenças estruturais entre esses dois pares tornam 2 e $\mathbf{5}$ mais apolares, portanto, esses compostos deveriam penetrar mais facilmente na barreira lipídica. Porém esses compostos ao penetrarem a barreira não devem causam nenhum efeito tóxico considerável à micobactéria o que pode ser comprovado por seus resultados de CIM inferior aos obtidos para 3 e 4. O que se observa a partir dos dados experimentais obtidos para essa classe de lignanas é que além do caráter hidrofóbico, relacionado à estrutura base desses compostos, existe a necessidade de uma hidroxila estar livre para maior atividade. Portanto, apesar do foco do trabalho não ser o mecanismo de ação dos compostos avaliados, pode-se, com base nos dados experimentais supor que a lipofilicidade é essencial para que essa classe de compostos consiga penetrar na barreira de lipídios das diferentes cepas, mas que para esse tipo de estrutura química a hidroxila livre é o que produz a toxicidade à micobactéria seja por desativação de enzimas, formação de radicais de oxigênio ativos ou alteração de metabolismo celular (Brunton et al., 2008; Harris et al., 2014)

Em um trabalho anterior relatamos a atividade antimicobacteriana de lignanas naturais e sintéticas, observando que essas atividades estão altamente relacionadas com o esqueleto químico destes compostos que podem ser lactonas, lactóis ou cetais (Silva et al., 2009). Comparandose os resultados de todas as classes de lignanas avaliadas por nosso grupo de pesquisa pode-se concluir que o esqueleto químico mais promissor é o dibenzilbutanodioico presente na diidrocubebina (1) e derivados 3-4 que, apesar da atividade moderada foi ativo contra duas cepas de Mycobacterium avaliadas $M$. tuberculosis e $M$. avium.

\section{CONCLUSÃO}

Apesar da moderada atividade antimicobacteriana apresentada, o esqueleto 
dibenzilbutanodioico pode ser um bom alvo na busca por novas moléculas bioativas através de modificação estrutural em 1 mantendo uma hidroxila livre ligada ao $\mathrm{C9}$ e alterando o substituinte em C9 '(R2) pela introdução de grupos com funções orgânicas, polaridade e tamanho diferentes.

\section{AGRADECIMENTOS}

Os autores agradecem a Fundação de Amparo à Pesquisa do Estado de São Paulo (FAPESP) e Conselho Nacional de Desenvolvimento Científico e Tecnológico (CNPq) pelo apoio financeiro.

\section{REFERÊNCIAS}

AKINYEMI, K.O. et al. Screening of crude extracts of six medicinal plants used in South-West Nigerian unorthodox medicine for anti-methicillin resistant Staphylococcus aureus activity. BMC Complementary Alternative Medicine, v.5, p.6-9, 2005.

ARBEX, M.A. et al. Drogas antituberculose: interações medicamentosas, efeitos adversos e utilização em situações especiais. Parte 1: fármacos de primeira linha. Jornal Brasileiro de Pneumologia, v.36, p.626640, 2010.

BADHEKA, L.P. et al. Lignans of Piper cubeba. Phytochemistry, v.26, p.2033-2036, 1987.

BRUNTON, L.; PARKER, K.; BLUMENTHAL, D.; BUXTON, I. Goodman \& Gilman's Manual of Pharmacology and Therapeutics, 1.ed. New York: McGraw Hill, 2007, $1219 p$.

CANTRELL, C.L. et al. Antimycobacterial plant terpenoids. Planta Medica, v.67, p.1-10, 2001.

CARVALHO, M.G. et al. Lignans from Nectandra turbacensis. Phytochemistry, v.26, p.265-267, 1987.

COLLINS, L.A.; FRANZBLAU, S.G. Microplate alamar blue assay versus BACTEC 460 system for high-throughput screening of compounds against Mycobacterium tuberculosis and Mycobacterium avium. Antimicrobial Agents and Chemotherapy, v.41, p.1004-1009, 1997.

DAMAYANTHI, Y.; LOWN, J.W. Podophyllotoxins: current status and recent developments. Current Medicinal Chemistry, v.5, p.205-252, 1998.

DECLOUR, A. Outer membrane permeability and antibiotic resistance. National Institutes of Health, v.1749, n.5, p.808-816, 2009.

DEY, D. et al. Antitubercular and antibacterial activity of quinonoid natural products against multi-drug resistant clinical isolates. Phytotheraphy Research., v.28, p.1014-1021, 2014.

DIACON, A.H. et al. Multidrug-resistant tuberculosis and culture conversion with bedaquiline. The New England Journal of Medicine, v.371, p.723-732, 2014.

DZIDIC, S. et al. Antibiotic resistance mechanisms in bacteria: Biochemical and genetic aspects. Food Technology Biotechnology. v.46, n.11, p.11-21, 2008. ELFAHMI, et al. Lignan profile of Piper cubeba, an Indonesian medicinal plant. Biochemical Systematics and Ecology, v.35, p.397-402, 2007.

GARCÍ, A. et al. Recent advances in antitubercular natural products. European Journal of Medicinal Chemistry, v.49, p.1-23, 2012.

GUIMARÃES, D.O. et al. Antibióticos: importância terapêutica e perspectivas para a descoberta e desenvolvimento de novos agentes. Química Nova, v.33, p.667-679, 2010.

GORDALIZA, M. et al. Podophyllotoxin distribution, sources, applications and new cytotoxic derivatives. Toxicon, v.44, p.441-459, 2004.

GU, J.Q. et al. Antitubercular constituents of Valeriana laxiflora. Planta Medica v.70, p.509-514, 2004.

HARDMAN, J.G.; LIMBIRD, L.E.; GILMAN, A.G. The Pharmacological Basic of Therapeutics, 10.ed. McGraw Hill Toronto, 2001, 2045p.

HARRIS, K.K. et al. Novel imidazoline antimicrobial scaffold that inhibits DNA replication with activity against mycobacteria and drug resistant gram-positive cocci. ACS Chemical Biology, v.9, n.11, p.2572-2583, 2014.

HERNANDEZ, J.D. et al. Ariensin, a new lignan from Bursera ariensis. Planta Medica, v.47, n.4, p. 215-217, 1983.

Korycka-Machala, M.; Rumijowska-Galewicz, A.; Dziadek, $J$. The effect of ethambutol on mycobacterial cell wall permeability to hydrophobic compounds. Polish Journal of Microbiology, v.54, p.5-11, 2005.

KOUL, A. et al. The challenge of new drug discovery for tuberculosis. Nature, v.469, p.483-490, 2011.

MOREIRA, R.R.D. et al. Paepalanthus spp: Antimycobacterial activity of extracts, methoxylated flavonoids and naphthopyranone fractions. Revista Brasileira de Farmacognosia, v.23, p.268-272, 2013.

MURRAY, P.R.; ROSENTHAL, K.S.; PFALLER, M.A. Medical Microbiology. 7.ed. Philadelphia: Elsevier, 2012, p960.

OKUNADE, A.L.; ELVIN-LEWIS, W.H. Natural antimycobacterial metabolites current status. Phytochemistry, v.65, p.1017-1032, 2004.

PALOMINO, J.C. et al. Resazurin microtiter assay plate simple and inexpensive method for detection of drug resistance in Mycobacterium tuberculosis. Antimicrobial Agents and Chemotherapy, v.46, p.2720-2722, 2002.

PISTOLOZZI, M. et al. Enantiomeric HPLC resolution and absolute stereochemistry assignment of a new poligamain derivative. Journal of Pharmaceutical and Biomedical Analysis, v.75, p.118-122, 2013.

RAVIGLIONE, M.C. The TB epidemic from 1992 to 2002. Tuberculosis, v.83, p.4-14, 2003.

RICCARDI, G.; PASCA, M.R. Trends in discovery of new drugs for tuberculosis therapy. The Journal of Antibiotics, v.67, p.655-659, 2014.

SARAIVA, J. et al. In vitro and in vivo activity of lignan lactones derivatives against Trypanosoma cruzi. Parasitology Research, v.100, p.791-793, 2007.

SARAIVA, J. et al. Cubebin and derivatives as inhibitors of mitochondrial complex I. Proposed interaction with subunit B8. Journal of Enzyme Inhibition and Medicinal Chemistry, v.24, p.599-606, 2009.

SILVA, M.L.A. et al. Antimycobacterial activity of natural and semi-synthetic lignans. Zeitschrift für Naturforschung C 64, p.779-784, 2009.

SILVA, R. et al. Synthesis and biological activity evaluation

Rev. Bras. PI. Med., Campinas, v.17, n.4, supl. I, p.782-789, 2015. 
of lignan lactones derived from (-) - cubebin. Bioorganic Medicinal Chemistry Letters, v.15, p.1033-1037, 2005.

SILVA, R. et al. Trypanocidal structure-activity relationship for cis- and trans-methylpluviatolide. Phytochemistry, v.69, p.1890-1894, 2008.

SILVA, M.L.A et al. Trypanosoma cruzi: evaluation of (-) - cubebin derivatives activity in the messenger RNAs processing. Parasitology Research, v.109, p.445-451, 2011.

SOUZA, V.A. et al. Trypanocidal activity of (-)-cubebin derivatives against free amastigote forms of Trypanosoma cruzi. Bioorganic Medicinal Chemistry Letters, v.15, p.303-305, 2005.

TIMMINS, G.S.; DERETIC, V. Mechanisms of action of isoniazid. Molecular Microbiology, v.62, p.1220-1227, 2006.

TIMPLE, J.M. et al. The lignan (-)-hinokinin displays modulatory effects on human monoamine and GABA transporter activities. Journal of Natural Products, v.76, n.10, p.1889-95, 2013.

USIA, T.; WATABE, T.; KADOTA, S.; TEZUKA, Y. Potent CYP3A4 inhibitory constituents of Piper cubeba. Journal of Natural Products, v.68, p.64-68, 2005.

VERG, E.; PLUMPE, G.; SCHULTHEIS, H. Milestones. The Bayer Story 1863-1988. West Germany: Leverkusen, 1988, 624p.

WALSH, C. Antibiotics: Actions, Origins, Resistence, 2.ed. Washington, DC: ASM Press, 2003, 345p.

WORLD HEALTH ORGANIZATION (WHO). World Tuberculosis Day. http://www.who.int/campaigns/tbday/2014/event/en/. Acesso em: 26 nov. 2014.

YAM, J. et al. Piper cubeba demonstrates anti-estrogenic and anti-Inflammatory properties. Planta Medica, v.74, p.142-146, 2008a.

YAM, J. et al. Piper cubeba targets multiple aspects of the androgen-signalling pathway. A potential phytotherapy against prostate cancer growth? Planta Medica, v.74. p.33-38, 2008b. 